\title{
ANALYTIC FUNCTIONS INTO BANACH SPACES AND A NEW CHARACTERIZATION FOR ISOMORPHIC EMBEDDINGS
}

\author{
V. WROBEL
}

\begin{abstract}
Let $T: E \rightarrow F$ be a continuous linear injection between complex Banach spaces. It is shown that $T$ is a topological monomorphism if and only if every function $f: D \rightarrow E$ on the open unit disc of the complex plane, for which $T \circ f$ is analytic, is itself analytic.
\end{abstract}

1. Introduction. In the paper [1, p. 292] the following problem was raised:

Let $f: D:=\{z \in \mathrm{C}:|z|<1\} \rightarrow l^{p}(1 \leqslant p<\infty)$ denote a function which is analytic when considered as a mapping into $l^{\infty}$. Does this imply that $f: D \rightarrow l^{p}$ itself is analytic?

The answer is no in general, as the following theorem of Globevnik [2, p. 75] shows.

THEOREM. Let $1 \leqslant p<\infty$. There exists a function $f: D \rightarrow l^{p}$ with the following properties.

(i) $f$ is not continuous;

(ii) $f$ is analytic in $D$ as a function into $l^{p^{\prime}}$ for any $p^{\prime}>p$;

(iii) the derivatives of $f$ (as a function into $l^{p^{\prime}}$ ) of all orders are functions from $D$ into $l^{p}$.

Globevnik [2, p. 76] states that this theorem also holds true for $L^{p}(S, \Sigma, \mu)$ and $L^{p^{\prime}}(S, \Sigma, \mu)\left(p^{\prime}>p\right)$ provided that $\Sigma$ contains a disjoint sequence $\left\{E_{n}\right\}$ of measure 1. Therefore it seems to be very natural to pose the following problem which generalizes $(+)$.

Let $T: E \rightarrow F$ denote a continuous linear injection between two complex Banach spaces $E$ and $F$. Give necessary and sufficient conditions for $T$ such that for every mapping $f$ : $D \rightarrow E$ we have $f$ is analytic if and only if $T \circ f$ is analytic.

An obvious sufficient condition in $(++)$ is that $T$ is a topological monomorphism. At the first glance it may seem surprising that this condition is also necessary (see Theorem 1 below), but an analysis of the facts underlying Theorem 1 (see §4) gives that it is not. The Globevnik result holds in fact for any two Banach spaces $E$ and $F$ such that $E$ is an algebraic but not a topological subspace of $F$.

Received by the editors October 19, 1981.

1980 Mathematics Subject Classification. Primary 30A99, 46A30, 46E40.

Key words and phrases. Banach spaces, analytic functions, isomorphic embeddings. 
2. Notations. Throughout this paper $D$ will denote the open unit disc of the complex plane. A mapping $f: D \rightarrow E$ into a complex locally convex space $E$ is said to be analytic, if $f$ can be represented locally by absolute convergent (with respect to the topology of $E$ ) power series expansions. If $M$ is a subset of a locally convex space $E$, let $\operatorname{cl}(M, E)$ denote the closure of $M$ in $E$. A continuous linear mapping $T$ : $E \rightarrow F$ between two locally convex spaces is not nearly open provided that there exists a neighborhood $U_{0}$ of zero in $E$ such that $\operatorname{cl}\left(T\left(U_{0}\right), T E\right)$ is not a neighborhood of zero in $T E$. All locally convex spaces are complex linear spaces.

\section{Main results.}

THEOREM 1. Let $T: E \rightarrow F$ be a continuous linear injection from a Banach space $E$ into a Fréchet space $F$. Then the following are equivalent.

(i) $T$ is a topological monomorphism;

(ii) if $f: D \rightarrow E$ is such that $T \circ f: D \rightarrow F$ is analytic, then $f$ itself is analytic.

REMARKS. (1) The implication “(i) implies (ii)" is easy and remains true for any two locally convex spaces $E$ and $F$ such that $T E$ is closed in $F$, whereas the converse is not true in the general locally convex setting. This can be seen very easily by letting $E$ be an infinite dimensional Banach space, letting $F$ be the same space but equipped with its weak topology $\sigma\left(E, E^{\prime}\right)$, and letting $T$ denote the identity mapping. Then (ii) holds by a Banach-Steinhaus argument, whereas (i) obviously fails.

(2) Theorem 1 also holds if $E$ and $F$ are two Fréchet spaces. Indeed, the proof of Theorem 1 was initially done in this setting using the techniques of Globevnik [2] and a result due to the author $[3$, p. 130] cinaracterizing non-nearly-open mappings into metrizable locally convex spaces. Later on it turned out that the proof of Theorem 1 as well as that of Globevnik's theorem can be significantly simplified when restricting the statement to the form in which Theorem 1 is given here. The reason why $F$ is allowed to be a Fréchet space nevertheless is that the normed case does neither simplify the statement nor the proof at all, and that the Fréchet space case seems to be interesting in itself, especially if compared with the first remark.

(3) If (i) is not true, we shall produce a discontinuous function $f: D \rightarrow E$ such that $T \circ f$ is analytic. Indeed, we shall prove the following result, which generalizes Globevnik's theorem.

THEOREM 2. Let $T: E \rightarrow F$ be a continuous linear injection from a Banach space $E$ into a Fréchet space $F$. If $T$ is not a topological monomorphism, then there exists a function $f: D \rightarrow E$ such that

(i) $f$ is not continuous;

(ii) $T \circ f$ is analytic;

(iii) the derivatives of $T \circ f$ of all orders are mappings from $D$ into $T E$.

As the proof of Theorem 2 will show, condition (i) can be replaced by

(i)' $f$ is not locally bounded. 
Hence in view of this observation, one may pose the following

Problem. Let $T: E \rightarrow F$ be a continuous linear injection from a Banach space $E$ into a Fréchet space $F$, and let $f: D \rightarrow E$ denote a locally bounded function such that $T \circ f$ is analytic. Does this already imply that $f$ is analytic itself?

There is a positive answer to this problem if $f$ is assumed to be measurable by a Cauchy formula argument as has been pointed out to the author by J. Voigt (München).

4. The proof of Theorem 1 and auxiliary results. We start with a result characterizing mappings which are not nearly-open from a normed space into a metrizable locally convex space. This result is a special form of Satz 1 in $[3$, p. 130].

LEMMA 1. Let $T: E \rightarrow F$ be a continuotus linear injection from a normed space $E$ into a metrizable locally convex space $\left(F,\left\{q_{n}\right\}\right)\left(\left\{q_{n}\right\}\right.$ denoting a generating sequence of semi-norms). Suppose that $T$ is not nearly-open. Then for every sequence $\left\{\gamma_{n}\right\}$ of positive numbers there exist sequences $\left\{x_{n}\right\}$ in $E$ and $\left\{x_{n}^{\prime}\right\}$ in $E^{\prime}$ such that

(i) $\left\|x_{n}\right\|=1, q_{n}\left(T x_{n}\right)<\gamma_{n}(n \in \mathrm{N})$,

(ii) $\left\|x_{n}^{\prime}\right\| \leqslant 2^{n-1}(n \in \mathbf{N})$,

(iii) $\left(x_{n}, x_{m}^{\prime}\right)=\delta_{n, m}(n, m \in \mathrm{N})$.

For the sake of completeness we give a proof. In order to do so we need the following result established in [3, p. 130].

Proposition 1. Let $E$ and $F$ denote two locally convex spaces, and let $T: E \rightarrow F$ be a continuous linear mapping. Suppose that $\mathrm{cl}\left(T\left(U_{0}\right), T E\right)$ is not a neighborhood of zero in $T E$ for some neighborhood of zero $U_{0}$ in $E$. Then for every closed subspace $M$ of $E$ with finite codimension $\mathrm{cl}\left(T\left(U_{0} \cap M\right), T M\right)$ is not a neighborhood of zero in $T M$.

Proof OF LEMMA 1. Without loss of generality we may assume that $q_{n} \leqslant q_{n+1}$ ( $n \in N)$. As $\operatorname{cl}(T(B(0,1)), T E)$, where $B(0,1)$ denotes the open unit ball centered at 0 , is not a neighborhood of zero in $T E$, we find $x_{1} \in E$ such that

$$
\left\|x_{1}\right\|=1 \text { and } q_{1}\left(T x_{1}\right)<\gamma_{1} \text {. }
$$

By the Hahn-Banach theorem we find $x_{1}^{\prime} \in E^{\prime}$ such that

$$
\left(x_{1}, x_{1}^{\prime}\right)=1 \text { and }\left\|x_{1}^{\prime}\right\|=1 .
$$

So let us assume that we constructed $x_{1}, \ldots, x_{n} \in E$ and $x_{1}^{\prime}, \ldots, x_{n}^{\prime} \in E^{\prime}$ such that

(i) $\left\|x_{k}\right\|=1, q_{k}\left(T x_{k}\right)<\gamma_{k}(1 \leqslant k \leqslant n)$,

(ii) $\left\|x_{k}^{\prime}\right\| \leqslant 2^{k-1}(1 \leqslant k \leqslant n)$,

(iii) $\left(x_{k}, x_{m}^{\prime}\right)=\delta_{k, m}(1 \leqslant k, m \leqslant n)$.

We choose

$$
x_{n+1} \in N_{n}:=\bigcap_{k=1}^{n} \operatorname{kern} x_{k}^{\prime}
$$

such that

$$
\left\|x_{n+1}\right\|=1 \text { and } q_{n+1}\left(T x_{n+1}\right)<\gamma_{n+1} \text {. }
$$


Such a choice is possible, by Proposition 1, because $\operatorname{cl}\left(T\left(B(0,1) \cap N_{n}\right)\right)$ is not a neighborhood of zero in $T N_{n}$. By means of the Hahn-Banach theorem we choose $x^{\prime} \in E^{\prime}$ such that

$$
1=\left(x_{n+1}, x^{\prime}\right) \text { and }\left\|x^{\prime}\right\|=1
$$

Then we define

$$
x_{n+1}^{\prime}:=x^{\prime}-\sum_{k=1}^{n}\left(x_{k}, x^{\prime}\right) x_{k}^{\prime}
$$

This gives

$$
\left(x_{k}, x_{n+1}^{\prime}\right)=\delta_{k, n+1} \text { and }\left\|x_{n+1}^{\prime}\right\| \leqslant 2^{n} .
$$

This completes the proof.

In order to prove Theorems 1 and 2 we need the following result which is essentially contained in the proof of Lemma 1 in $[2$, p. 73].

LEMMA 2. Let $1 \leqslant p<\infty$, and let $\beta_{n}>0(n \in \mathrm{N})$. Then there exists a sequence of polynomials $\left\{p_{n}\right\}$ such that

(i) $\left\{p_{n}^{(k)}(z)\right\} \in l^{p}$ for every $z \in D$ and $k \in \mathbf{N}_{0}$;

(ii) there exists a sequence $\left\{z_{n}\right\}$ in $D$ converging to $2^{-1}$ such that $\left|p_{n}\left(z_{n}\right)\right| \geqslant \beta_{n}$.

The proof of this lemma is done in the same way as the construction in the proof of Globevnik's Lemma 1 in [2, p. 73], where $\beta_{n}=n(n \in \mathrm{N})$.

Proof OF THeOREMS 1 AND 2. Let $\beta_{n}:=n 2^{n-1}(n \in N), p=1$ and apply Lemma 2. This gives us a sequence of polynomials $\left\{p_{n}\right\}$ with properties (i) and (ii). Next choose

$$
\gamma_{n}:=\left(3^{2 n} \max \left(1, \sup \left\{\left|p_{n}^{(k)}(z)\right|: k \in \mathbf{N}_{0}, z \in D\right\}\right)\right)^{-1} \quad(n \in \mathbf{N})
$$

in Lemma 1. Hence we obtain sequences $\left\{x_{n}\right\}$ in $E$ and $\left\{x_{n}^{\prime}\right\}$ in $E^{\prime}$ with properties (i)-(iii). Then we define $f: D \rightarrow E$ by

$$
f(z):=\sum_{n=1}^{\infty} p_{n}(z) x_{n} \in \operatorname{cl}\left(\operatorname{span}\left\{x_{n}: n \in \mathbf{N}\right\}, E\right)
$$

(the convergence is pointwise and absolute by the choice of $\left.\left\{x_{n}\right\}\right)$. By the choice of $\left\{x_{n}^{\prime}\right\}$ the mapping $A: E \rightarrow l^{\infty}$ defined by $A x:=\left\{\left(x, 2^{-n+1} x_{n}^{\prime}\right)\right\}$ is continuous (and linear). But the function $A \circ f: D \rightarrow l^{\infty}$ is not locally bounded at $2^{-1}$. Indeed,

$$
\left\|A \circ f\left(z_{n}\right)\right\|=\sup \left\{\left|\left(\sum_{l=1}^{\infty} p_{l}\left(z_{n}\right) x_{l}, 2^{-k+1} x_{k}^{\prime}\right)\right|: k \in \mathbf{N}\right\} \geqslant n
$$

by the choice of the sequence $\left\{p_{n}\right\}$. Thus $f: D \rightarrow E$ is not continuous. Next let $B$ : $l^{\infty} \rightarrow l^{1}$ be defined by $B\left(\left\{\xi_{n}\right\}\right)=\left\{2^{n-1} \gamma_{n} \xi_{n}\right\}$. This again is a continuous linear mapping, and by construction $B \circ A \circ f: D \rightarrow l^{1}$ given by $B \circ A \circ f(z)=\left\{\gamma_{n} p_{n}(z)\right\}$ is locally bounded and hence analytic by the Aron-Cima theorem [1]. Finally $C\left(\left\{\eta_{n}\right\}\right):=\sum_{n=1}^{\infty} \gamma_{n}^{-1} \eta_{n} T x_{n}$ defines a continuous linear mapping from $l^{1}$ into $F$. Therefore $T \circ f=C \circ B \circ A \circ f$ is analytic in $D$, whereas $f$ is not. The additional 
property (iii) in Theorem 2 follows from the construction, which gives that $\left\{p_{n}^{(k)}(z)\right\}$ $\in l^{1}\left(z \in D, k \in \mathbf{N}_{0}\right)$ and $q_{n}\left(T x_{n}\right) \rightarrow 0$. This completes the proof.

ACKNowledgement. The author wants to express his gratitude to Professor Floret for suggesting the problem $(++)$ mentioned in the introduction and for making several valuable remarks on the text. Thanks are also due to Dr. J. J. Koliha for several fruitful talks.

\section{REFERENCES}

1. R. Aron and J. A. Cima, A theorem on holomorphic mappings into Banach spaces with basis, Proc. Amer. Math. Soc. 36 (1972), 289-292.

2. J. Globevnik, On analytic functions into IP-spaces, Proc. Amer. Math. Soc. 61 (1976), 73-76.

3. V. Wrobel, Streng singuläre Operatoren in lokalkonvexen Räumen, Math. Nachr. 83 (1978), 127-142.

Mathematisches Seminar der Universitxt, Olshausenstrasse 40-60, D-2300 Kiel 1, Federal REPUBlic OF GERMANY 